\title{
AVALIAÇÃO DO COMPORTAMENTO CARDIOVASCULAR, DA MOBILIDADE FUNCIONAL E MARCHA DE HEMIPARÉTICO CRÔNICO EM ESTEIRA ERGOMÉTRICA
}

Carla de Oliveira Carletti, Thiago Kendy Kawano, Vanessa Niens Van Den Broek, Fabiana Araújo Silva, Lúcia Martins Barbatto, Augusto Cesinando de Carvalho

Universidade Estadual Paulista - UNESP, Departamento de Fisioterapia, Presidente Prudente, SP.

\section{RESUMO}

O objetivo do presente estudo foi avaliar o comportamento cardiovascular, a mobilidade funcional e marcha de um individuo hemiparético crônico submetido a um protocolo de treinamento aeróbio em esteira ergométrica sem suporte de peso corporal. Estudo de caso com um indivíduo hemiparético crônico, avaliado por meio do Timed up and go (TUG), Teste de Velocidade de Caminhada de $10 \mathrm{~m}$ (TV10M) e Teste de Esforço (TES). Foi submetido a um protocolo de treinamento em esteira ergométrica de 30 min divididos em 3 blocos de 10 min cada, com intervalos de descanso, em uma velocidade de $60 \%$ daquela obtida no TES. Não foi observado alteração no comportamento da frequência cardíaca e da pressão arterial ao longo do tratamento, todavia observou-se melhora no TUG ( $A V 1=12,53 \mathrm{seg}$; AV2 = 9,39 seg) e do TV10M (AV1 = $9,00 \mathrm{seg} ; \mathrm{AV} 2=7,03 \mathrm{seg}$ ). Os resultados demonstraram que o protocolo utilizado não o foi suficiente para alterar a condição cardiovascular de um individuo hemiparético crônico submetido a protocolo de treinamento aeróbio em esteira ergométrica sem suporte de peso corporal, todavia melhorou a condição motora funcional.

Palavras-chave: acidente vascular cerebral, hemiparesia, marcha, exercício, fisioterapia.

\section{EVALUATION OF CARDIOVASCULAR BEHAVIOR, FUNCTIONAL MOBILITY AND GAIT IN CHRONIC HEMIPARETIC TREADMILL}

\begin{abstract}
The aim of this study was to evaluate the cardiovascular behavior, functional mobility and gait of a chronic hemiparetic individual subjected to an aerobic training protocol on a treadmill without body weight support. Case study with a chronic hemiparetic individual assessed by the Timed Up and Go (TUG), Walking Speed Test $10 \mathrm{~m}$ (TV10M) and Stress Testing (TES). It underwent a training protocol on a treadmill $30 \mathrm{~min}$ divided into three 10 -min blocks each, with rest intervals, at a speed of $60 \%$ of that obtained in TES. was not observed change in behavior of the heart rate and blood pressure during the treatment, however there was improvement in TUG (AV1 $=12.53 \mathrm{sec} ; A V 2=9.39 \mathrm{sec})$ and TV10M (AV1 = $9.00 \mathrm{sec} ; A V 2=7.03 \mathrm{sec})$. The results showed that the protocol used was not sufficient to alter the cardiovascular condition of a chronic hemiparetic individual subjected to aerobic training protocol on a treadmill without body weight support, but improved functional motor condition.
\end{abstract}

Keywords: stroke, hemiparesis, march, exercise, physiotherapy.

\section{INTRODUÇÃO}

O acidente vascular Cerebral (AVC) é caracterizado como uma doença cerebrovascular que pode ocorrer na forma isquêmica, causado por uma interrupção súbita do fluxo sanguíneo através da obstrução de uma artéria do encéfalo ou por uma ruptura que caracteriza o AVC hemorrágico ${ }^{1}$. É considerada a principal causa de incapacidade neurológica, pois gera impacto em diversas funções humanas, constituindo um problema de saúde pública por ser uma das maiores causas de morte no mundo e pelos altos custos de seu tratamento ${ }^{2}$. Dentre as manifestações clínicas, estão os prejuízos das funções sensitivas, motoras, de equilíbrio e de marcha, além do déficit cognitivo e de linguagem ${ }^{3}$. 
A hemiparesia é a disfunção motora predominante nos indivíduos que sofreram AVC e representa uma redução do controle motor no hemicorpo contralateral a lesão encefálica. Além disso, as principais deficiências apresentadas pelos indivíduos com hemiparesia são redução da força e da resistência muscular, alteração do tônus muscular, déficit de coordenação da marcha, assimetria na distribuição de peso, entre outras $^{3}$. Todas essas alterações interferem no desempenho das atividades de vida diária, sendo as disfunções da marcha os principais fatores agravantes de limitação funcional ${ }^{3,4}$.

A maioria dos indivíduos que sofreram AVC apresenta uma diminuição na capacidade aeróbia e na capacidade de gerar força em relação a indivíduos saudáveis com a mesma idade $^{5}$.

A reabilitação do paciente com sequela de AVC envolve primariamente a fisioterapia convencional, entretanto, outras técnicas foram sugeridas recentemente para melhorar a capacidade de suportar esforços, o desempenho da marcha e a capacidade funcional de forma geral, além de diminuir a pressão arterial (PA) e os fatores de risco cardiovasculares ${ }^{3}$. Alguns exemplos incluem a caminhada em esteira, com ou sem suspensão de peso corporal, o treinamento aeróbio e o fortalecimento muscular ${ }^{6}$.

O treinamento cardiovascular realizado na esteira ergométrica deve ser definido a partir da porcentagem da frequência cardíaca máxima (FCmax), de reserva (FCR) ou VO2max ${ }^{7,8}$.Valores entre $20-39 \%$ da FCR determinam um treinamento leve, enquanto que $40-59 \%$ moderado e $60-84 \%$ forte ${ }^{9}$. Dessa forma, estudos revelam que a intensidade da reabilitação convencional é baixa, com menos de $30 \%$ da FCR, contribuindo para baixos níveis de aptidão cardiovascular. Portanto, o aumento da intensidade dos exercícios durante a reabilitação após o AVC pode ser considerado benéfico ${ }^{10,11}$.

Diante disso, o objetivo do presente estudo foi avaliar o comportamento cardiovascular, a mobilidade funcional e marcha de um individuo hemiparético crônico submetido a um protocolo de treinamento aeróbio em esteira ergométrica sem suporte de peso corporal.

\section{METODOLOGIA}

Para realização deste estudo foi recrutado indivíduo hemiparético frequentador de um centro de atendimento de Fisioterapia e Reabilitação em Presidente Prudente. Os critérios de inclusão do estudo foram: indivíduo com hemiparesia unilateral decorrente ao AVC com encaminhamento médico apresentando tempo de lesão $\geq$ há 12 meses; marcha com ou sem auxílio de órtese ou meio auxiliar de locomoção e habilidade para realizar teste de caminhada, já os critérios de exclusão do estudo foram: dupla hemiparesia, tempo de lesão inferior a 12 meses, presença de déficits cognitivos avaliados pelo Mini-exame do Estado Mental (ponto de corte para indivíduos analfabetos 20 e para indivíduos com diferentes anos de instrução escolar 25-29), afasia sensitiva ou condições de saúde adversas, tais como outras doenças neurológicas ou ortopédicas não relacionadas ao AVC. O voluntário foi informado sobre os objetivos e procedimentos do estudo e após concordar com sua participação, assinou um termo de consentimento livre e esclarecido. $O$ estudo foi aprovado pelo Comitê de Ética da FCT-UNESP (CAAE: 34601014.4.0000.5402).

$\mathrm{O}$ individuo hemiparético foi submetido a uma entrevista individual para coleta de dados clínicos, características antropométricas, verificação dos critérios de inclusão, sendo posteriormente realizada avaliação inicial (AV1) utilizando testes motores funcionais 1 - Timed up and go $(T U G)^{12}$ para avaliar a mobilidade funcional durante a marcha, 2- Teste de Velocidade de Marcha de 10 metros (TV10M) ${ }^{13}$ avaliar a velocidade da marcha, 3- Teste de Esforço (TES) para verificar a tolerância do indivíduo ao exercício e à fadiga. Todos os testes foram repetidos após 7 semanas na avaliação final (AV2).

Após as avaliações o indivíduo iniciou o protocolo de treinamento que consistiu em 30 minutos, divididos em três blocos de dez minutos cada, de caminhada na esteira ergométrica a uma velocidade de $60 \%$ da encontrada no TES. Os intervalos entre os blocos foram determinados pelo tempo necessário para que a FC retornasse ao valor inicial de repouso. Durante estes intervalos, o indivíduo permaneceu sentado em uma cadeira posicionada sobre a esteira ergométrica. A FC foi monitorada durante todo o treinamento por meio da observação de um monitor cardíaco portátil POLAR RS800cxR. Os valores da FC foram expressos em batimentos 
cardíacos por minuto (BPM). A PA foi aferida com o indivíduo na postura ortostática, no início e final de cada bloco. Também se utilizou a Escala Modificada de Borg (EMB), através de pergunta direta e demonstração da tabela no início da caminhada, no quinto minuto e após o fim de cada bloco. $O$ treinamento foi realizado duas vezes na semana, com duração de 30 minutos por sessão durante sete semanas, totalizando 14 sessões.

\section{RESULTADOS}

A amostra foi constituída por um indivíduo de 57 anos de idade, hipertenso, com diagnóstico clínico de AVC isquêmico há 1 ano, com hemiparesia espástica à direita. Em todas as sessões, o paciente realizou os três blocos de treinamento completando o tempo total de 30 minutos.

A Tabela 1 apresenta os valores da pressão arterial sistólica e diastólica de repouso e ao final do treinamento das 14 sessões.

Tabela 1. Valores da pressão arterial sistólica e diastólica de repouso e ao final do treinamento das 14 sessões (médias \pm DP).

\begin{tabular}{lcc}
\hline Condição & Pressão arterial & $\mathbf{m m H g}$ \\
\hline Repouso & Sistólica & $135 \pm 7,59$ \\
& Diastólica & $77 \pm 6,11$ \\
\hline Final & Sistólica & $136 \pm 6,33$ \\
& Diastólica & $85 \pm 5,18$ \\
\hline
\end{tabular}

Os resultados dos testes motores funcionais realizados em AV1 e AV2 encontramse na Tabela 2.

Tabela 2. Resultados dos testes funcionais para avaliar a marcha.

\begin{tabular}{l|c|c}
\hline Teste & AV1 & AV2 \\
\hline TES (Km/h) & 3,88 & 3,82 \\
\hline TUG (seg) & 12,53 & 09,39 \\
\hline TV10M (seg) & 9,00 & 07,03 \\
\hline
\end{tabular}

TES: Teste de Esforço Máximo; TUG: Timed up and go; TV10M: Teste de Velocidade de $10 \mathrm{~m}$.

$\mathrm{Na}$ Tabela 3, encontram-se os valores da Escala Modificada de Borg (EMB) ao final de cada bloco da 1a sessão e da 14a sessão de treinamento.
Tabela 3. Valores da Escala Modificada de Borg (EMB) ao final de cada bloco da 1a sessão e da 14a sessão de treinamento.

\begin{tabular}{lcc}
\hline Blocos & $\begin{array}{c}\text { EMB final } \\
\left(1^{\text {a }} \text { sessão }\right)\end{array}$ & $\begin{array}{c}\text { EMB final } \\
\left(14^{\mathrm{a}} \text { sessão }\right)\end{array}$ \\
\hline 1 & 12 & 12 \\
2 & 11 & 12 \\
3 & 12 & 12 \\
\hline
\end{tabular}

$\mathrm{Na}$ Tabela 4, observa-se o intervalo da FCR e as médias e desvios padrões da FC inicial e final de cada bloco das 14 sessões de treinamento.

Tabela 4. Valores da frequência cardíaca $(F C)$ inicial e final de cada bloco das 14 sessões de treinamento (médias $\pm \mathrm{DP}$ ).

\begin{tabular}{lcc}
\hline Blocos & Condição & FC (bpm) \\
\hline 1 & Inicial & $73,28 \pm 4,30$ \\
& Final & $80,14 \pm 7,09$ \\
\hline 2 & Inicial & $73,28 \pm 4,30$ \\
& Final & $83,5 \pm 7,03$ \\
\hline 3 & Inicial & $73,28 \pm 4,30$ \\
& Final & $87,64 \pm 6,02$ \\
\hline Valores de & $40 \%$ & 102 \\
FCR & $69 \%$ & 131 \\
\hline
\end{tabular}

FCR: Frequência Cardíaca de Reserva - valores estabelecidos pela American College of Sport Medicine - ACMS para treinamento de baixa a moderada intensidade.

\section{DISCUSSÃO}

No presente estudo não foi observado alteração no comportamento da frequência cardíaca e da pressão arterial ao longo do tratamento, todavia observou-se melhora no TUG $(A V 1=12,53 \mathrm{seg} ; A V 2=9,39 \mathrm{seg})$ e do TV10M (AV1 = 9,00 seg; $A V 2=7,03 \mathrm{seg})$. O TES não apresentou alteração importante, bem como a EMB.

Indivíduos portadores de sequela de AVC apresentam um alto custo energético durante a realização das atividades em especial da marcha, podendo contribuir para a fadiga precoce, dispneia, depressão, ansiedade, estilo de vida sedentário e consequentemente descondicionamento físico ${ }^{5}$. Dessa forma, a esteira ergométrica pode ser utilizada como recurso fisioterapêutico para intervir nos comprometimentos físicos e na qualidade de vida após o AVC. 
Apesar das evidências dessas alterações após o AVC, poucos são os estudos que tem investigado os efeitos do treinamento aeróbio na capacidade funcional e na qualidade de vida desses indivíduos. Além disso, os protocolos de treinamento aeróbio expostos na literatura apresentam grande diversidade em relação à frequência e duração, o que dificulta a comparação dos resultados encontrados e a elaboração de um consenso 5 .

Entretanto, sabe-se que à intensidade do treinamento aeróbio em esteira ergométrica para pacientes hemiparéticos deve ser individualizada, pois depende das respostas subjetivas (taxa de percepção de esforço) e objetivas (FC e PA) de cada um.

De acordo com Kuys et al. ${ }^{11}$ o treinamento em esteira realizado com intensidades entre $40 \%$ e $59 \%$ da FCR resulta na melhora da capacidade de caminhar e aptidão cardiovascular de indivíduos hemiparéticos quando comparados com a terapia convencional. No presente estudo, a velocidade da esteira não desencadeou alteração na $F C$, PA e no EBM, o que se pode levar em consideração a velocidade calculada para a esteira ( $60 \%$ do TES), pois diante disto o individuo não entrou na faixa de treinamento da FCR o que consequentemente não melhorou o desempenho cardiovascular, embora houvesse melhora da velocidade da marcha determinada pelos testes motores funcionais. Assim, acreditase que com o aumento da velocidade da esteira o comportamento cardiovascular poderia ser sido alterado.

No entanto, intensidades fortes entre 60$84 \%$ da FCR para indivíduos hemiparéticos leva-se em consideração alguns estudos que abordam que o treino de marcha na esteira pode aumentar o padrão de marcha anormal favorecendo a assimetria corporal ${ }^{11}$.

Segundo dados da literatura, a duração de um programa de exercícios aeróbios deve ser de pelo menos oito semanas, para se obter um efeito positivo sobre os parâmetros cardiovasculares $^{11}$. Macko et al. ${ }^{14}$ pôde verificar esse dado em seu estudo, realizando um programa de treinamento aeróbio em esteira com duração de seis meses em indivíduos com sequela de AVC na fase crônica sendo observado poucos ganhos funcionais até o terceiro mês do programa, fator este também que leva-se em consideração para os resultados apresentados no presente estudo, pois não foram realizadas oito semanas.
Dessa forma, sugerem-se novos estudos sobre protocolos de treinamento aeróbio em esteira ergométrica sem suporte de peso corporal em indivíduos hemiparéticos crônicos, no intuito de melhorar a condição cardiovascular, mobilidade funcional e marcha. Outros protocolos com maior tempo de intervenção devem ser pesquisados bem como outros delineamentos experimentais (maior amostra e grupo controle) já que estes pacientes apresentam descondicionamento físico devido às alterações no controle motor diminuindo sua capacidade funcional.

\section{CONCLUSÃO}

O presente estudo demonstrou que o protocolo utilizado não o foi suficiente para alterar a condição cardiovascular de um individuo hemiparético crônico submetido a protocolo de treinamento aeróbio em esteira ergométrica sem suporte de peso corporal, todavia melhorou a condição motora funcional.

\section{CONFLITOS DE INTERESSE}

Os autores declaram não haver qualquer potencial de conflito de interesse que possa interferir na imparcialidade deste trabalho científico.

\section{REFERÊNCIAS}

1. Grochovsk CS, Campos RC, Lima MCAM. Ações de Controle dos Agravos à Saúde em Indivíduos Acometidos por Acidente Vascular Cerebral. Rev Bras Ciênc Saúde. 2015;19(4):269-76. DOI: https://doi.org/10.4034/RBCS.2015.19.04.03

2. Lucena EMF, Morais JD, Batista HRL, Mendes LM, Silva SQR, Neves RF et al. A funcionalidade de usuários acometidos por AVE em conformidade com a acessibilidade à reabilitação. Acta Fisiatr. 2011;18(3):112-8.

3. Lima AP, Cardoso FB. O Efeito de um Programa de Exercícios Físicos sobre a Capacidade Funcional da Marcha Hemiparética de Indivíduos com Acidente Vascular Cerebral. Rev Bras Ciênc Saúde. 2014;18(3):203-8. DOI: https://doi.org/10.4034/RBCS.2014.18.03.03

4. Natalio MA, Nunes GS, Herber $V$, Michaelsen SM. Relação entre cadência da subida e descida de escada, recuperação motora e equilíbrio em indivíduos com hemiparesia. Acta Fisiatr. 2011;18(3):146-50.

5. Moura RMF, Lima RCM, Lage DC, Amaral EAA. Efeitos do treinamento aeróbio na qualidade de vida e na capacidade funcional de indivíduos 
hemiparéticos crônicos. Acta Fisiatr. 2005;12(3):94-99.

6. Ovando AC, Michaelsen SM, Dias JA, Herber V. Treinamento de marcha, cardiorrespiratório e muscular após acidente vascular encefálico: estratégias, dosagens e desfechos. Fisioter Mov. 2010;23(2):253-69.

DOI: https://doi.org/10.1590/S0103-

\section{9}

7. Garber CE, Blissmer B, Deschenes MR, Franklin BA, Lamonte MJ, Lee IM, et al. American College of Sports Medicine position stand. Quantity and quality of exercise for developing and maintaining cardiorespiratory, musculoskeletal, and neuromotor fitness in apparently healthy adults: guidance for prescribing exercise. Med Sci Sports Exerc. 2011;43(7):1334-59. DOI: https://doi.org/10.1249/MSS.0b013e318213fefb

8. Askim T, Dahl AE, Aamot IL, Hokstad A, Helbostad J, Indredavik B. High-intensity aerobic interval training for patients 3-9 months after stroke: a feasibility study. Physiother Res Int. 2014;19(3):129-39.

DOI: https://doi.org/10.1002/pri.1573

9. Mezzani A, Hamm LF, Jones AM, McBride PE, Moholdt T, Stone JA, et al. Aerobic exercise intensity assessment and prescription in cardiac rehabilitation: a joint position statement of the European Association for Cardiovascular Prevention and Rehabilitation, the American Association of Cardiovascular and Pulmonary Rehabilitation and the Canadian Association of Cardiac Rehabilitation. Eur J Prev Cardiol. 2013;20(3):442-67. DOI: https://doi.org/10.1177/2047487312460484 10. MacKay-Lyons MJ, Makrides L. Cardiovascular stress during a contemporary stroke rehabilitation program: Is the intensity adequate to induce a training effect? Arch Phys Med
Rehab.

2002;83(10):1378-83.

DOI: https://doi.org/10.1053/apmr.2002.35089

11. Kuys SS, Brauer SG, Ada L. Higher-intensity treadmill walking during rehabilitation after stroke in feasible and not detrimental to walking pattern or quality: a pilot randomized trial. Clin Rehabil. 2011;25(4):316-26. DOI: https://doi.org/10.1177/0269215510382928

12. Podsiadlo D, Richardson S. The timed "Up \& Go": A test of basic functional mobility for frail elderly persons. J Am Geriatr Soc. 1991;39(1):142-8. DOI: https://doi.org/10.1111/i.15325415.1991.tb01616.x

13. Bowden MG, Balasubramanian CK, Behrman AL, Kautz SA. Validation of a Speed-Based Classification System Using Quantitative Measures of Walking Performance Post-Stroke. Neurorehabil Neural Repair. 2008;22(6):672-5. DOI: https://doi.org/10.1177/1545968308318837 14. Macko RF, Ivey FM, Forrester LW, Hanley D, Sorkin JD, Katzel LI, et al. Treadmill exercise rehabilitation improves ambulatory function and cardiovascular fitness in patients with chronic stroke: a randomized, controlled trial. Stroke. 2005;36(10):2206-11. DOI: https://doi.org/10.1161/01.STR.0000181076.918 $\underline{05.89}$

Recebido para publicação em 17/08/2016

Revisado em 12/09/2016

Aceito em 15/09/2016 